\title{
$30 \mathrm{MHz}$ ultrasonic attenuation of TiNi single crystal associated with B2-B19' martensitic transformation and twin boundaries
}

\author{
X. Ren, X.L. Liang ${ }^{1}$, K. Otsuka ${ }^{2}$, T. Suzuki ${ }^{3}$, H.M. Shen ${ }^{1}$ and Y.N. Wang ${ }^{1}$ \\ Materials Physics Group, National Institute for Materials Science, Tsukuba, Ibaraki 305-0047, \\ Japan \\ ${ }^{1}$ Department of Physics, Nanjing University, Nanjing, China \\ ${ }^{2}$ Smart Structure Research Center, National Institute of Advanced Industrial Science \\ and Technology, Tsukuba, Japan \\ ${ }^{3}$ Tsukuba Institute of Science and Technology, Tsukuba, Japan
}

\begin{abstract}
Ultrasonic attenuation was measured for the first time at megahertz range (30MHz) for a TiNi single crystal, which undergoes B2-B19' (cubic-monoclinic) martensitic transformation. A sharp attenuation peak was found at transformation temperature region (two-phase region), which was associated with the structural transformation and drastic domain wall evolution in the two-phase region. Besides, a very broad peak below the transformation temperature (i.e., in martensite state) was also found, which can be ascribed to twin boundary motion at megahertz range. This broad peak has a high internal friction level of 0.0027 . Considering that this alloy also has a high damping capacity at low frequency, we can deduce that TiNi martensite is an excellent high damping material in an extremely wide frequency range and wide temperature range.
\end{abstract}

\section{INTRODUCTION}

Damping capacity is the capability of a material to absorb mechanical vibration (i.e., changing mechanical energy into thermal energy). Compared with usual metals and alloys, martensitic alloys exhibit extraordinary high damping capacity (about two order of magnitude higher) in transformation temperature region and in martensitic state. This high damping capacity is generally regarded as due to the motion of martensite/parent phase interface and due to the motion of martensite domain walls (twin boundaries) [1-4]. Such a process dissipates mechanical energy and generates a high damping capacity. The damping in transformation region is mainly associated with the amount of transformation within one cycle of vibration [1].

The high damping capacity of martensitic alloys has attracted considerable attention over the past decades, and they are promising candidates for wide applications [5].Despite the fact there have been many studies on internal friction of martensitic alloys, there still remain some unclear questions and issues. The first question is to how high a frequency the high damping capacity can persist. To our knowledge almost all the experiments so far were performed with low frequency torsion pendulum or cantilever method. The frequency is limited mostly to $100 \mathrm{~Hz}$. Recent study [4] on kilohertz range revealed some new interesting features: even the damping peak associated with the transformation is frequency dependent.

Another unclear issue is the origin of the broad damping peak in martensitic state. The martensite damping peak has been reported in various ferroelastic/martensitic materials, such as TiNi [6,7], AuCd [8], LNPP [9], etc. There is no doubt that this peak is associated with domain wall motion, because this peak disappears if using single domain martensite [9]. However, the nature of the peak still remains unclear. Recent study suggested that this peak may be related to the interaction of point defects with martensite domain walls [10].

In view of these unsolved issues, it becomes necessary to expand damping experiment to megahertz range. In the present paper, we report our preliminary results on a $\mathrm{Ti}_{50} \mathrm{Ni}_{50}$ single crystal measured at $30 \mathrm{MHz}$. This experiment, combined with a series of on-going experiments, may be able to give some new insight into these problems. The use of single crystal can rule out additional effect due to grain boundary and thus provide clearer information.

\section{EXPERIMENTAL}

TiNi single crystals were prepared with modified Bridgman method with nominal composition of $\mathrm{Ti}_{50} \mathrm{Ni}_{50}$. The crystals were grown at Tomsk physical technical institute by Prof. Chumlyakov's group. 
The single crystal ingot was spark-cut into a parallelepiped shape with surface normal $\langle 100\rangle$, followed by appropriate polishing by sandpaper. Then the sample was sealed in quartz tube filled with argon gas, and treated at $1273 \mathrm{~K}$ for 1 hour, followed by ice-water quench. The sample was slightly polished to remove surface oxidation during quench. The size of the sample was $6.4 \times 3.8 \times 2.8 \mathrm{~mm}^{3}$.

In fact, we measured elastic constants of this sample in parent state with rectangular parallelepiped resonance method [11], in an attempt to study the precursor elastic softening phenomenon.

The transformation temperatures were determined by DSC measurement of the same sample. This alloy is known to undergo a B2-B19' transformation, where $\mathrm{B} 19^{\prime}$ martensite has an unusual monoclinic non-basal structure [12]. The cooling exothermic peak was shown in Fig.1a, where the cooling rate is $5 \mathrm{~K} / \mathrm{min}$. Transformation temperatures were determined to be $\mathrm{Ms}=276 \mathrm{~K}, \mathrm{Mf}=246 \mathrm{~K}$, $A s=289 \mathrm{~K}, \mathrm{Af}=314 \mathrm{~K}$. The transformation temperature of the sample seems lower compared the known value of this alloy (Ms is around $323 \mathrm{~K}$ ). This may be due to the use of graphite crucible that interacts with $\mathrm{Ti}$ of the alloy, resulting in a depletion of $\mathrm{T}^{\mathrm{i}}$ in the alloy. Thus transformation temperature is lowered.

Ultrasonic attenuation was measured by pulse-echo overlapping method at $30 \mathrm{MHz}$ with [001] longitudinal wave. The measurement was carried out during cooling from B2 parent phase to martensite. Due to the limitation of our cryostat, we can go down only to about $240 \mathrm{~K}$. Our ongoing experiment will try to reach a lower temperature.

$\mathrm{Ti}_{50} \mathrm{Ni}_{50}$ single crystal

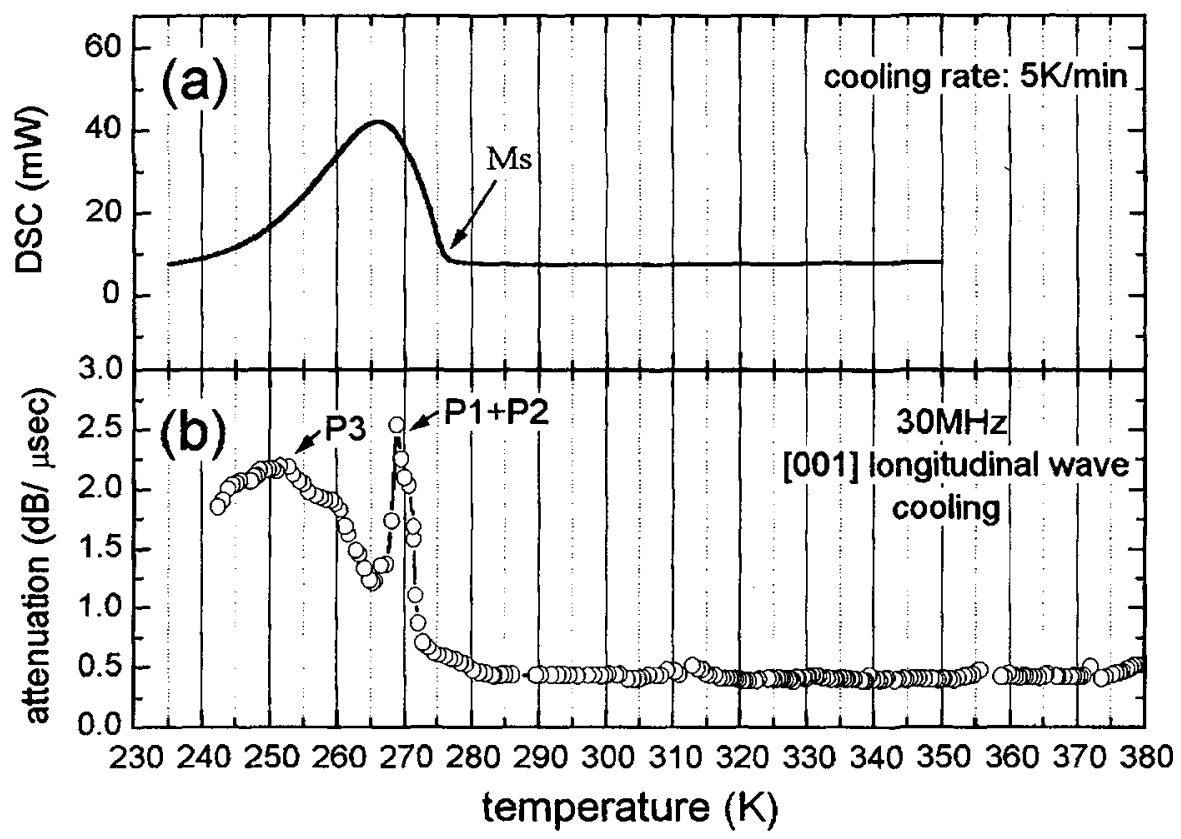

Figure 1: $30 \mathrm{MHz}$ ultrasonic attenuation in a Ti50Ni50 single crystal as a function of temperature

\section{RESULTS}

\subsection{Sharp peak associated with B2-B19' transformation}

In Fig. $1 \mathrm{~b}$, we can see that there are two peaks at $268 \mathrm{~K}$ and $252 \mathrm{~K}$, respectively. The position of $268 \mathrm{~K}$ peak nearly coincides with the DSC peak temperature (266K). Considering that DSC measurement uses a higher cooling rate than attenuation measurement, one can deduce that the difference is mainly due to the different cooling rate. Therefore, we can conclude that the $268 \mathrm{~K}$ peak is due to the B2-B19' martensitic transformation. We assign this peak as $\mathrm{P} 1+\mathrm{P} 2$, and the reason will be given later. A more detailed discussion will be given in discussion section. 


\subsection{Broad peak associated with martensite domain walls}

The second peak appears at $252 \mathrm{~K}$, at which martensitic transformation have almost finished. This peak is very broad; it is very similar to the previously reported broad twin peak P3 at $200 \mathrm{~K}$ under low frequency $(\sim 1 \mathrm{~Hz})$ measurement condition [6,7], therefore, we tentatively assign this peak as P3. It should be noted that early studies ascribed this peak as due to dislocations $[6,7]$, but there is no critical proof of it in metallic systems. On the other hand, systematic study on inorganic ferroelastic LNPP crystal obtained convincing evidence that this peak is due to domain walls of the low symmetry phase (martensite) [9], because this peak disappears when using single-domain martensite.

Then if this peak is $P 3$, we can deduce that this peak is shifted from $200 \mathrm{~K}$ to $252 \mathrm{~K}$ when frequency changes from the order of $1 \mathrm{~Hz}$ to $30 \mathrm{MHz}$. We are performing experiment at different frequencies to obtain the exact frequency dependence of this peak over a wide frequency range in order to see if the behavior can be explained by available theories.

\subsection{Premartensitic attenuation}

From Fig. $1 \mathrm{~b}$ we found attenuation begins to increase at about $283 \mathrm{~K}$, which is $7 \mathrm{~K}$ above Ms temperature. We believe that this may be related to the premartensitic heterophase fluctuation, which can dissipate some vibration energy and causes attenuation. However, the magnitude seems too small. This may be due to the fact that we used longitudinal [001][001] waves, which interact very little with the transverse strain fluctuation $(<110><-110>$ shear). To confirm this, we have also observed the temperature dependence of the resonance peaks of this sample by rectangular parallelepiped resonance method measured at $100 \sim 1000 \mathrm{kHz}$ range [13]. As a result, we found that attenuation begins to increase at least $50 \mathrm{~K}$ above Ms temperature [13]. Our on-going work will try to use $\langle 110><-110\rangle$ transverse wave to study the premartensitic attenuation.

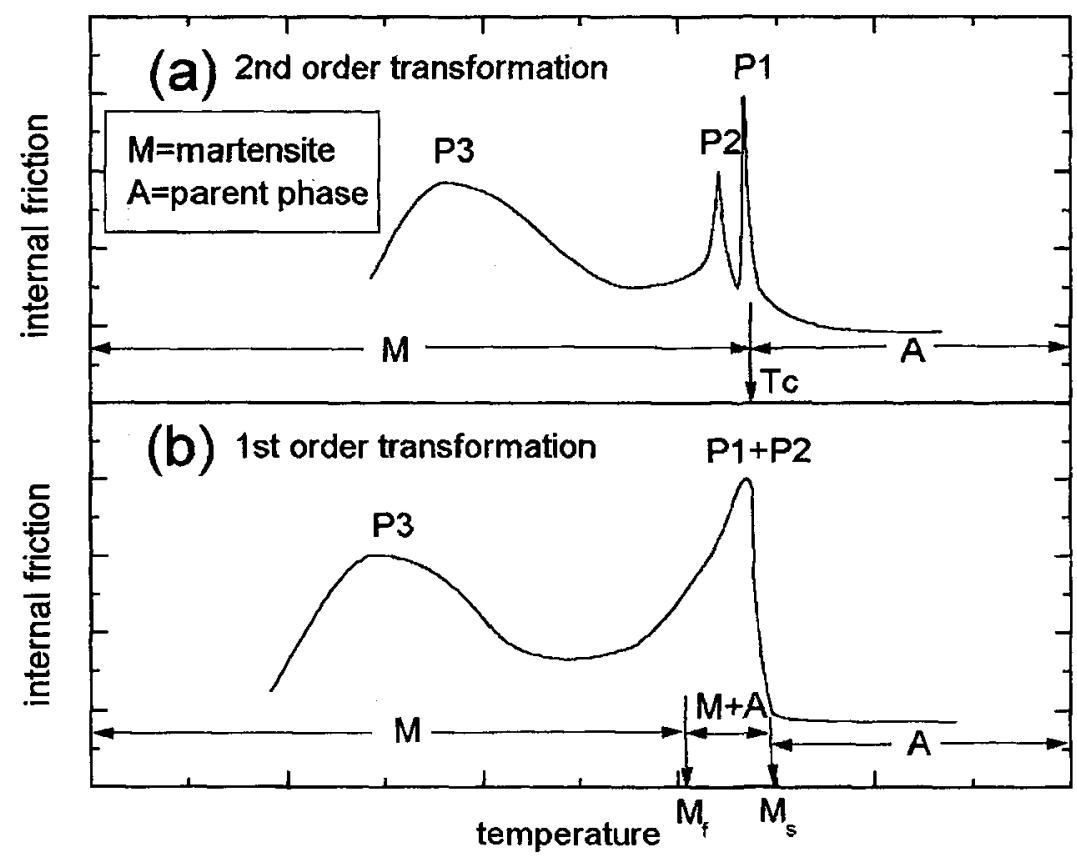

Figure 2: Schematic internal friction as a function of temperature for (a) second order ferroelastic transformation and (b) first order ferroelastic/martensitic transformation. 


\section{DISCUSSION}

\subsection{Damping peaks associated with $2^{\text {nd }}$ order and $1^{\text {st }}$ order ferroelastic/martensitic transformation and domain wall motion}

Internal friction associated with $2^{\text {nd }}$ order ferroelastic transformation has been systematically studied in Wang and her coworkers [9]. Their results are schematically shown in Fig.2a. For comparison, internal friction associated with $1^{\text {st }}$ order ferroelastic/martensitic transformation is shown in Fig. $2 \mathrm{~b}$, which is based on a large number of previous publications [6-8].

There are three internal friction peaks for materials undergoing $2^{\text {nd }}$ order ferroelastic transformation, as shown in Fig.2a. P1 is related to the fluctuation of order parameter (strain), which reaches maximum at $\mathrm{Tc}$; it also coincides with the condensation of related shear modulus or phonon [9]. This strain fluctuation can interact with external stress thus causes energy dissipation. P2 is proved to be due to the coarsening of domains with lowering temperature [9]; this is a result of increasing interaction between domains due to the increase of order parameter with lowering temperature. P3 is also related to domain walls, but the mechanism is not very clear. Huang et al. [10] suggested that it is due to a "collective pinning of domain walls by point defects".

For $1^{\text {st }}$ order transition, the picture is similar to the $2^{\text {nd }}$ order case, but with some differences, as shown in Fig.2b. The P3 peak, which is related to domain walls, is the same as the $2^{\text {nd }}$ order transformation case. The mechanism is expected to be the same as $\mathrm{P} 3$ in the $2^{\text {nd }}$ order case, which requires clarification. For $1^{\text {st }}$ order ferroelastic/martensitic transformation, transformation generates two-phase interfaces which can be considered as a source for a damping peak (here we call P1). Besides there is drastic martensite domain evolution during martensite formation (which is similar to the domain coarsening of the $2^{\text {nd }}$ order transformation case), which also produces a damping peak (we call P2). Because $1^{\text {st }}$ order martensitic transformation occurs in a relatively wide temperature interval, from Ms to Mf (which is typically $10 \sim 30 \mathrm{~K}$ for most shape memory alloys). Then both processes concur in this two-phase region; thus in most cases we can see only one peak and thus it is assigned as P1+P2. For weakly $1^{\text {st }}$ order transformation $\mathrm{P} 1$ and $\mathrm{P} 2$ peaks are partially separated and one may see a "shoulder (P1)" on the high temperature side of P2 peak. This situation has indeed been observed in In-Tl alloys [14], which undergoes a nearly $2^{\text {nd }}$ order transformation.

The most popular mechanism for the $\mathrm{P} 1+\mathrm{P} 2$ peak is the aforementioned interface (including martensite/parent interface and martensite twin interface) effect. This mechanism, if true, requires that damping should begin to increase at Ms temperature at which interfaces begin to appear. However, our study [13] at a few hundred kilohertz range found that damping begins to increase at least $50 \mathrm{~K}$ above Ms. This cannot be explained by this mechanism. The present study also finds a small attenuation increase $7 \mathrm{~K}$ above Ms temperature. We expect that the use of shear waves may detect the attenuation increase at higher temperature, if the nature of this premartensitic attenuation is related to the heterophase fluctuation. Further work is being done towards this direction.

\subsection{Using broad $P 3$ peak to realize high damping capacity over wide temperature and frequency range}

From the attenuation value of broad peak P3 (at $252 \mathrm{~K}$ ), we can calculate the corresponding internal friction at $30 \mathrm{MHz}$ is a high level of 0.0027 . Because we know that this broad damping peak also exists even at very low frequencies (but shifted to $200 \mathrm{~K}$ ) [6,7], we can reach an important conclusion: we can use this broad peak to realize high damping capacity over a wide temperature region (i.e., 100K) and over an extremely wide frequency range.

$\mathrm{P} 1+\mathrm{P} 2$ peak is also a candidate for high damping applications; but this peak is strongly dependent on cooling/heating rate, because this peak is mainly due to the transient part of internal friction, which vanishes if measuring at constant temperature. Therefore, P3 is more suitable for a stable high damping capacity.

Because most applications require high damping capacity around room temperature, it is necessary to shift the $\mathrm{P} 3$ peak to around room temperature. This can be obtained by adjusting transformation temperature to around $373 \mathrm{~K}\left(100^{\circ} \mathrm{C}\right)$ through alloying addition so as to move the broad $\mathrm{P} 3$ peak to around room temperature. This is a simple way to obtain high damping capacity at arbitrary 
temperature region, and it is also an advantage over other types of high damping materials, such as the amorphous-hydrogen high damping materials (which is not easy to significantly change the damping peak position).

\section{SUMMARY}

1. Our ultrasonic attenuation experiment on single crystal TiNi showed that martensitic alloys have high damping capacity (loss tangent 0.0027 ) even up to $30 \mathrm{MHz}$ in martensite state. Therefore, this kind of materials is an excellent candidate for high damping applications of extremely wide frequency range (from $\mathrm{OHz}$ up to megahertz region) and wide temperature region.

2. Even at megahertz range, the temperature dependence of attenuation shows essentially the same feature as low frequency measurement. There are two peaks; one is associated with the B2-B19' transformation (which involves drastic evolution of phase boundaries and martensite twin boundaries), and the other is associated with martensite twin boundary motion. However, it seems that the latter peak (called P3 peak) was significantly moved from $\sim 200 \mathrm{~K}$ (when frequency is a few $\mathrm{Hz}$ ) to $250 \mathrm{~K}$ (when frequency is $30 \mathrm{MHz}$ ).

3. There seems a small attenuation increase prior to martensitic transformation. It is consistent with our observation of the temperature dependence of the resonance peaks. Further study is necessary to sensitively detect the precursory attenuation by using $<110<<-110>$ shear waves.

\section{Acknowledgments}

We are grateful to Prof. Yu. I. Chumlyakov at Tomsk Physical Technical Institute for growing single crystals, and to Dr. M. Asai at Yokohama R \&D Laboratory, the Furukawa Electric Co. Ltd. for providing TiNi ingots. This work was supported by "Research for the Future Program" of Japan Society of Promotion of Sciences (JSPS) under the category of High Damping Materials (1998-2002).

\section{Reference}

1. Y.N. Wang, Z.J. Yang, H. Zhu, J.C. Ma, J. Nanjing Univ., 7, 1(1963).

2. J.W. Christian, J. Inst. Met., 98, 546(1965).

3. J. Van Humbeek and L. Delaey, in B.R. Roth, M.S. Misra (Eds.) Proc. Int. Conf. "Role of Interfaces on Material Damping", Toroto, ASM, USA, 1985, P.59.

4. Y.N. Huang, Y.N. Wang, H.M. Shen, Phys. Rev. B46, 3290(1992).

5. J. Van Humbeek and Y. Liu, Mater. Sci. Forum, 327-328, 331(1999).

6. R. Hasiguti and K. Iwasaki, J. Appl. Phys. 59, 3128(1968).

7. J.S. Zhu, R. Schaller and W. Benoit, Phys. Lett. A, 141, 177 (1989).

8. Y. Murakami, K. Otsuka, H. Mizubayashi, T. Suzuki, Proc. Int. Conf. on Displacive Phase Transformations and Their Applications in Materials Engineering. TMS, (1998), $p$

9. Y.N. Wang, W.Y. Sun, X.H. Chen, H.M. Shen, B.S. Lu, Phys. Stat. Sol. (a), 102, 279(1987).

10. Y.N. Huang, X. Li, Y. Ping, Y.N. Wang, H.M. Shen, Z.F. Zhang, Phys. Rev. B55, 16159 (1997).

11. X. Ren, N. Miura, J. Zhang, K. Otsuka, K. Tanaka, M. Koiwa, T. Suzuki, Yu.I. Chumlyakov, M. Asai, Mater. Sci. Eng. A213, 196 (2000).

12. X. Ren and K. Otsuka, Scripta Mater., 38, 1669(1998).

13. X. Ren et al. unpublished work.

14. M. E. De Morton, J. Appl. Phys. 40, 208(1969). 